\title{
Proton dynamics in bacterial spores, a neutron scattering investigation
}

\author{
Alexandre Colas de la Noue ${ }^{1, a}$, Judith Peters ${ }^{2,3}$, Patrick Gervais ${ }^{1}$, Nicolas Martinez ${ }^{2,3}$, Jean-Marie Perrier-Cornet ${ }^{1}$ \\ and Francesca Natali ${ }^{2,4, b}$ \\ ${ }^{1}$ AgroSup Dijon-Université de Bourgogne, UMR PAM ,1 esplanade Erasme, 21000 Dijon, France \\ ${ }^{2}$ Institut Laue-Langevin, 71 avenue des Martyrs, CS 20156, 38042 Grenoble Cedex 9, France \\ ${ }^{3}$ Univ. Grenoble Alpes, IBS, 38044 Grenoble, France \\ ${ }^{4}$ CNR-IOM, OGG, 71 avenue des Martyrs, CS 20156, 38042 Grenoble Cedex 9, France
}

\begin{abstract}
Results from first neutron scattering experiments on bacterial spores are reported. The elastic intensities and mean square displacements have a non-linear behaviour as function of temperature, which is in agreement with a model presenting more pronounced variations at around $330 \mathrm{~K}\left(57^{\circ} \mathrm{C}\right)$ and $400 \mathrm{~K}\left(127^{\circ} \mathrm{C}\right)$. Based on the available literature on thermal properties of bacterial spores, mainly referring to differential scanning calorimetry, they are suggested to be associated to main endothermic transitions induced by coat and/or core bacterial response to heat treatment.
\end{abstract}

\section{Introduction}

When deprived of nutrients, Bacillus and Clostridia bacteria can form endospores which are metabolically inactive and can remain in this dormant state for years. Besides their ability to survive to starvation, bacterial spores are also highly resistant to various forms of stress such as heat, radiation or toxic chemicals $[1,2]$. Because of these exceptional properties, they are involved in food spoilage, foodborne diseases, they could be a threat as biological weapons, and might represent a probable vehicle for transfer of life between planets [3].

From the most external layers, bacterial spores are composed of an exosporium (absent in Bacillus subtilis), a proteinaceous layer called the coat, the outer membrane, the cortex mainly composed of a thick peptidoglycan structure, a cell wall, and the inner membrane, all surrounding the protoplasm or core [2] (Fig. 1). The latter contains the genetic material, protected by chaperone proteins called small acid-soluble proteins (SASPs) [4]. It is characterized by low water content $(0.6 \mathrm{~h}$, where $\left.\mathrm{h}=\mathrm{g}_{\text {water }} / \mathrm{g}_{\text {dry matter }}\right)^{3}$, an elevated concentration of ions and a complex of dipicolinic acid (DPA) and $\left(\mathrm{Ca}^{2+}\right)^{2}$. DPA is accumulated in high quantity in the core up to $10-15 \%$ of spore dry weight [5], largely beyond its solubility and it is involved in the dehydration of the core. The multi-resistance of the spores is the consequence of various protective strategies involving, but not limited to, the detoxication through external layer of the spore (i.e. the coat) $[1,2]$, a reduced inner membrane permeability (inner membrane) [6], and the protection of core content through a restriction of molecular mobility $[3,7,8]$. The first study addressing the immobility of proteins in the core was performed using Flurorescence Recovery After Photobleaching (FRAP) microscopy on a green fluorescent protein [7] (GFP) expressed exclusively in the spore core. The authors showed that this protein was immobile on the time-scale of a second even after the release of the large DPA depot. The role of the latter on the mobility and the protection of the core macromolecules is still not well understood. Recently, Sunde and co-workers [3] investigated the physical state of water in bacterial spores by using Nuclear Magnetic Resonance (NMR). They found that the water mobility in the core was quite similar as in binary protein water systems at the same hydration level (rotational correlation time of water $\sim 50 \mathrm{ps}$ ). However, the hydration of the core $(\sim 0.6 \mathrm{~h})$ did not fully explain the extreme heat resistance of bacterial spores. Therefore they suggested that the dormancy and resistance was not due to a quenching of molecular diffusion, but rather to a hindrance of protein rotation, probably as a consequence of the low core hydration. Asides the core peculiar properties, external layers also play an important role in spore resistance. Coat is implied in the resistance towards many chemicals and enzymes, and the cortex is involved in the resistance to wet heat, through the maintenance of protoplast low water content [2,9]. Sahin and co-workers [10] described the spore as a flexible structure which, in order to accomplish its function, has to expand and contract in response to humidity. This volume change was mostly attributed to the contraction of the cortex peptidoglycan $[11,12]$, less cross-linked than its vegetative counterpart [13]. Peptidoglycan typically shows viscoleastic properties: glassy polymer when dried and rubbery polymer when wet [14]. Even if organized in dense cross linked layers, the swelling of bacterial spore implies the spore coat being flexible. Finally, the core is also protected by the reduced permeability of the inner membrane [3], where lipids are largely immobile [6], and possibly in a gel phase [15].

Incoherent neutron scattering is a useful tool for investigating the properties of biological materials. In the late 80's, the technique permitted to establish the existence of a so-called dynamical transition [16], which 


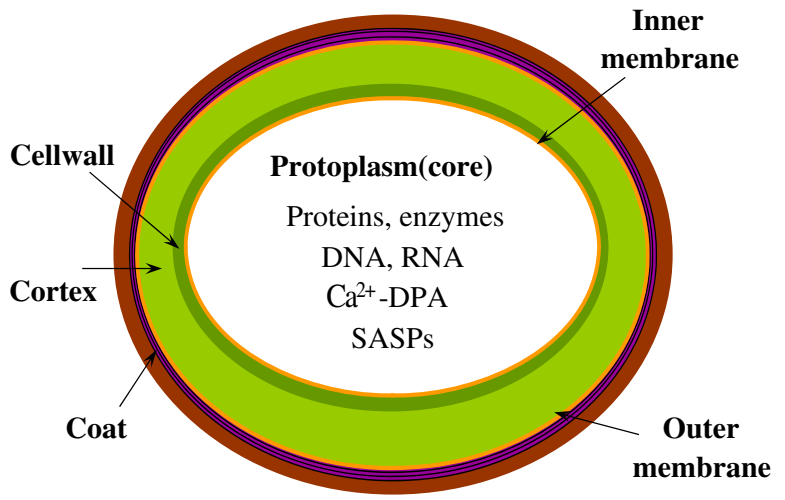

Figure 1. Bacillus subtilis spore structure (adapted from [1]).

has also been found by Mössbauer [17] and optical spectroscopy [18]. These findings allowed establishing a relation between structure, dynamics and function of biological systems. In the past two decades, incoherent neutron scattering was mainly used to characterize the dynamics of various protein systems and surrounding water $[19,20]$. The resolution of neutron experiments allows to monitor molecular motions from picosecond to nanosecond time scales. Some examples of recent advances related to biomolecules and dynamics could be: investigations of the influence of the amino acids side chains, the backbone [21] and the polypeptide chain [22] on the dynamical transition, the relation between secondary structure of proteins and dynamics [23], the influence of light excitation on the dynamics of photosensitive systems [24], the role of interfacial water on protein dynamics [25] and of the variation of water's density in the vicinity of hydrophilic or hydrophobic biomolecules [26]. The latest trends aim to study more and more complex systems, as cells in vivo [27], lipoproteins [28], plants' seeds [29] and brain tissue [30]. As illustrated in this introduction, the bacterial spore is a complex multi-layered structure. Roughly, it can be separated into two main compartments where molecular dynamics are supposed to differ significantly: the outer one (coat and cortex), and the inner one (inner membrane and protoplasm). Whereas the first one probably exhibits dynamics almost similar to the one observed for macromolecules, molecular movements of the second one are supposed to be confined. In the present study we investigated the dynamical properties of entire bacterial spores by neutron scattering, in order to compare the dynamics observed on the picosecond time-scale to results extracted from other physical measurements. The main objective was to provide a better understanding of their uncommon resistance properties due to the peculiarities of the core. We used a temperature range that progressively led to the complete inactivation of the spore population. This procedure allowed us to follow the dynamics of the spore components along with major structural changes, notably originating from the core deep rearrangement.

\section{Material and methods}

Spore samples were prepared from the Bacillus subtilis strain PS533 [31]. They were sporulated in liquid $2 *$ SG medium at $37^{\circ} \mathrm{C}$ and purified as described earlier [32]. The samples were free of growing cells, germinated cells and debris as observed by phase contrast microscopy (>98\%). Spore suspensions were stocked at $4{ }^{\circ} \mathrm{C}$ in distilled water until drying as described below. The bacterial spores in $\mathrm{H}_{2} \mathrm{O}$ were dehydrated in a ventilated chamber over lithium bromide saturated salt solution $(\sim 6 \%$ relative humidity $(\mathrm{RH})$ ) for at least 20 hours, then rehydrated by adsorption over pure $\mathrm{D}_{2} \mathrm{O}$ until a final content of $0.39 \mathrm{~h}$ dried spores. Total sample mass of $\sim 190 \mathrm{mg}$ was achieved. Following this procedure residual $\mathrm{H}$-water did not exceed $0.05 \mathrm{~h}$ and was therefore negligible in the scattering signal. The drying treatment did not affect spore viability.

Elastic incoherent neutron scattering (EINS) measurements as a function of temperature were performed on the thermal $(\lambda=2.23 \AA)$ high-energy resolution backscattering spectrometer IN13 (Institut Laue-Langevin, Grenoble, France) [33], characterized by a very large momentum transfer range $\left(0.2<\mathrm{Q}<4.9 \AA^{-1}\right)$ with a good and nearly Q-independent energy resolution ( $8 \mu \mathrm{eV}$ FWHM). IN13, therefore, allows accessing the space and time windows of $1-6 \AA$ and $0.1 \mathrm{~ns}$, respectively.

The elastic scattering intensities $\left(\mathrm{I}_{\mathrm{el}}(\mathrm{Q})=\mathrm{S}(\mathrm{Q}, \omega \approx\right.$ $0)$ ), properly corrected for the empty sample holder signal, were normalized with respect to a vanadium scan (typically used as a standard), to compensate for spurious background contributions and detector efficiency. From elastic incoherent measurements, it is possible to extract atomic mean square displacements (MSD), which represent the sample's flexibility at a given temperature, and the effective force constant that is a measure of protein resilience [34]. The signal essentially arises from the scattering on hydrogen atoms, as the incoherent scattering cross section of $\mathrm{H}$ is much bigger than for any other type of atoms present in biological samples. As the $\mathrm{H}$ atoms are moreover almost homogeneously distributed over the sample, neutron scattering probes the whole averaged molecular dynamics. The MSD were calculated from the slope of the elastic intensity at low Q (0.5$1.67 \AA^{-1}$, reported as solid lines in Fig. 2) following the approximation of the scattering law: $\mathrm{I}(\mathrm{Q}) \sim \mathrm{I}_{0} \exp (-$ $\left.\mathrm{MSD}^{*} \mathrm{Q}^{2} / 6\right)$, valid in the range of $\mathrm{Q}$ satisfying the Gaussian approximation $[16,35]$.

The sample mass and thickness were suitably chosen to optimize between a good signal-to-noise ratio and multiple scattering contributions (transmission of $96 \%$ in a $1.5 \mathrm{~mm}$ thick sample holder). To make the flat aluminum sample holder of dimensions $30 \times 40 \mathrm{~mm}^{2}$ vacuum tight at such high temperatures, a silicon seal (Motorsil paste) was used. It was oriented at $135^{\circ}$ with respect to the incoming neutron beam.

\section{Results}

In Fig. 2 we report the temperature dependence of elastic intensities of hydrated powder of wild bacterial spores over a wide temperature range (180-430 K). For clarity, only few selected temperatures are shown. Acquisition times between 90 and $180 \mathrm{~min}$ per $\mathrm{T}$ point were necessary to reach a good signal-to-noise ratio. Temperature was raised 


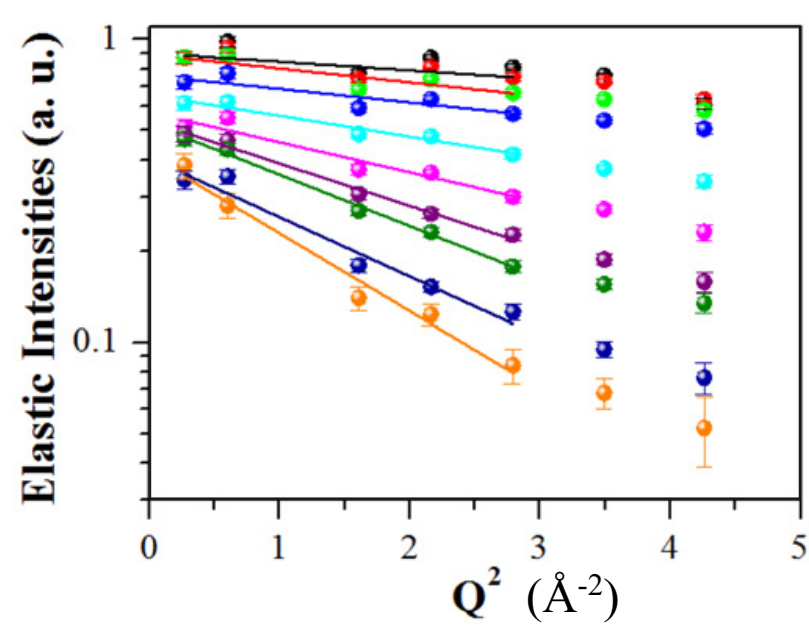

Figure 2. Temperature dependence of the elastic intensities of hydrated powder of bacterial spores over a wide temperature range $(180-430 \mathrm{~K})$. For clarity, only few selected temperatures are shown: black $=180 \mathrm{~K}$; red $=200 \mathrm{~K}$; green $=220 \mathrm{~K}$; blue $=$ $250 \mathrm{~K} ;$ cyan $=290 \mathrm{~K} ;$ pink $=327 \mathrm{~K}$; purple $=355 \mathrm{~K}$; dark green $=373 \mathrm{~K}$; dark blue $=400 \mathrm{~K}$; orange $=418 \mathrm{~K}$. Solid lines represent the slopes of the elastic intensities.

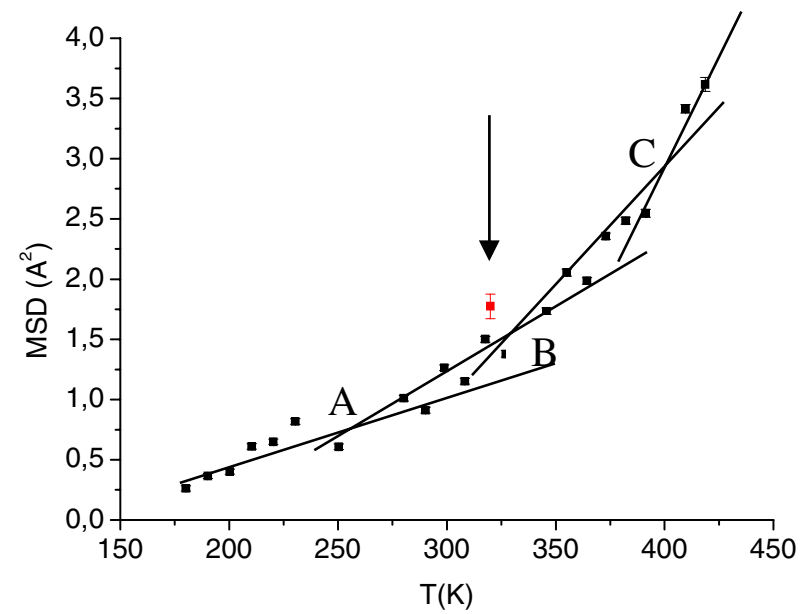

Figure 3. Temperature dependence of MSD extracted from the Q-range between 0.5 and $1.67 \AA^{-1}$ of hydrated powder of bacterial spores. The lines are guides to the eyes. The black arrow corresponds to the MSD after dropping the temperature from 420 to $320 \mathrm{~K}$.

stepwise (typically by $10 \mathrm{~K}$ ) followed by 5 minutes of equilibration time at each temperature point.

As the temperature increases the elastic intensities drop significantly, indicating considerable enhancement of proton dynamics. The unique $\mathrm{Q}$ range available on IN13 allows following the decreasing behaviour of the intensities up to 2 orders of magnitudes (y log scale in Fig. 2). The figure clearly shows that the acquisition time was chosen such that in the low Q range the statistical uncertainties are rather small. Presenting the intensities on a logarithmic scale, the linearity in Q2 is still visible until the highest temperature, and therefore the use of the Gaussian approximation is justified.

Figure 3 reports the MSD calculated for the hydrated spores in the low Q-range between 0.5 and $1.67 \AA^{-1}$.
The curve increases non-linearly over the temperature range investigated here. Based on previous knowledge we suggest distinguishing three regimes, indicated here by the intersection points between two straight lines, as the data seem consistent with such a model. The variations of the slope are signatures of changes in the spore local dynamics and in the spore's resilience.

Based on the extended literature focused on enhanced protein/membrane flexibility driven by solvent dynamics, the first break in slope (indicated as A and placed between 250 and $280 \mathrm{~K}$ ) can eventually be assigned to changes induced by water melting or to a shift of the dynamical transition to higher temperature. Contributions arising exclusively from water dynamics can be neglected here as the sample powders were hydrated in $\mathrm{D}_{2} \mathrm{O}$. The additional change in slope at $330-340 \mathrm{~K}$ (indicated as B) is more peculiar and will be discussed below.

To investigate eventual memory effect (hysteresis), once the spores have reached the final high temperature $(420 \mathrm{~K})$, the temperature was dropped to $320 \mathrm{~K}$ where a control acquisition run was performed (black arrow in Fig. 3). It is clear that the system was not able to recover its original behavior as confirmed by the slightly higher MSD indicating enhanced proton dynamics. A check of the sample after the experiment showed that no spores remained viable after the thermal slope. This was not surprising regarding the elevated temperature (max $420 \mathrm{~K}$ ) and acquisition time (180 min at high temperature). Variations in proton dynamics at high temperature will be discussed further, focusing on the major rearrangements of the spore's structure that follow heat inactivation [36].

\section{Discussion}

As explained in the introduction, bacterial spores are complex systems made of an inner core described as rigid body. The stiffness of the core is guaranteed by the dehydrated state of the spore interior with the specific role to protect it from heating $[2,3,37]$. The core is surrounded by several shells at different degrees of mobility.

The dynamical transition typically observed at $\mathrm{T} \sim$ $200-220 \mathrm{~K}$ in proteins at hydration state higher than $\mathrm{h} \sim$ $0.2^{16}$ was not resolved here. However, it is a matter of fact that the transition was shifted to higher temperature in more complex systems as natural membranes [38,39] or natural nanoparticles [22], but also in carbohydrates such as amylopectin or proteins when hydration was below $0.2 \mathrm{~h}[40,41]$. In consequence, it sensibly depends on the local environment and water content. The presence of a transition between 250 and $280 \mathrm{~K}$ might thus be a hint for water melting or for a shift of the dynamical transition due to a lowly hydrated environment. This would confirm the suggested idea of immobilized network of proteins in the core $[3,7]$. It could also reflect in part the amorphous solid state of Ca-DPA [5]. This finding should be related to the proposition of Sunde et al. [3] that core proteins are surrounded by at most one water layer. Our results suggest that core protein hydration is probably much lower (i.e. $<0.2 \mathrm{~h}$ ). The interpretation remains, however, difficult, because the hydration of $0.4 \mathrm{~h}$ of our spore might not be homogeneous in the inner and outer structure. The hydration level was chosen to avoid 
freezing of water at low temperature. As fully hydrated

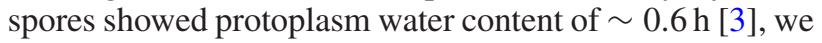
did not want to exceed $0.4 \mathrm{~h}$.

Why external layers (i.e. coat, cortex and cell wall) did not undergo a transition at $220 \mathrm{~K}$ remains unclear. No physical barrier exists for water diffusion within outer structures and they contain more water than the core in fully hydrated conditions [3]. The hydration level of the outer structure was not expected to be below $0.39 \mathrm{~h}$. Even if the coat proteins are highly cross linked and are expected to be rotationally immobilized [3], the dynamical transition was not only related to protein structure [22] and local motions of individual amino acids in the coat might not be restricted. Thus, one would have expected to observe a dynamical transition for the outer structures. We suggest that it was hidden by the stronger scattering intensity of the core, which exhibits a reduced macromolecular mobility.

Regarding the transition observed at 330-340 K, it should be associated to the endothermic transition at around $330 \mathrm{~K}$ observed by differential scanning calorimetry (DSC) measurements of bacterial spores [4244]. Formerly, it was interpreted in terms of a polymer glass-transition [45]. In this framework, the spores would remain in their dormant glassy state until melting and energy adsorption would occur at the endothermic transition temperature. This idea was also supported through complementary DSC and NMR investigations on Bacillus subtilis spores $[43,46]$. However, the exact origin of this transition remains unclear and the existence of a glassy state is still controversial [3]. The absence of a transition at low temperature $(200-220 \mathrm{~K})$ reminds the behavior of protein/trehalose glass in this temperature range [47] and shows that a glassy state could be considered for the protoplasm of bacterial spores.

Several works reported additional transitions in dormant B. subtilis at around 383 and $398 \mathrm{~K}$ [43-45], in agreement with feature $\mathrm{C}$ revealed here, but the origin of this transition is still not clearly understood. In our case, the transition observed around $400 \mathrm{~K}$ could be attributed to the radical change of the spore core dynamical properties as a consequence of the lethal heat treatment such as DPA leakage, core rehydration and protein denaturation [36]. The latter has also been shown to increase flexibility on Escherichia coli cells after a lethal heat treatment in an elastic neutron scattering experiment [27]. Transitions associated with thermal denaturation were also observed on various proteins systems such as small beta proteins [48] and apocalmodulin [49]. Receveur and co-workers [50] noted an increase of the hydrogen diffusing fractions and a slight increase of the sphere of diffusion in denaturated yeast phosphoglycerate kinase as compared with the native form. Thus the increased flexibility observed on bacterial spores around $400 \mathrm{~K}$ was probably a consequence of the progressive raise of core protein mobility following rehydration, and subsequent denaturation of immobilized core proteins. However, it could also come partly from the coat proteins denaturation, as a transition was observed with DSC measurements [44]. The memory effect shown in Fig. 3 could reflect the slow reversibility ( $\sim 5$ days) of the spore thermal properties observed with DSC for the 330-340 K transition [42]. However, it would rather be attributed to the way out of confinement of the core after being heat-killed. An increase of macromolecules' dynamics associated with the swelling of the core and the rehydration of macromolecules [5] after the lethal heat treatment could indeed explain the larger MSD observed at $320 \mathrm{~K}$.

\section{Conclusion}

The present study clearly shows the potential of neutron scattering to investigate such complex samples as spores. Neutrons are non-charged particles and permit to go deeply into the different compartments and to extract information about changes in molecular dynamics. EINS measurements give overall information about the variations in flexibility. The various microenvironment existing in the spore structure represents the main limitation. These experimental barriers can be partly overcome by using mutants lacking some layers/components. This approach allows to probe more accurately the structure, and to understand the role of specific components on spore's dynamics. Such experiments were also performed within the last year and data analysis is under progress.

\section{References}

[1] Setlow, P. J. Appl. Microbiol. 2006, 101, 514-525

[2] Leggett, M. J.; McDonnell, G.; Denyer, S. P.; Setlow, P.; Maillard, J. Y. J. Appl. Microbiol. 2012, 113, 485498

[3] Sunde, E. P.; Setlow, P.; Hederstedt, L.; Halle, B. Proc. Natl. Acad. Sci. USA 2009, 106, 19334-19339

[4] Setlow, P. Trends Microbiol. 2007, 15, 172-180

[5] Kong, L.; Setlow, P.; Li, Y.-Q. Analyst. 2012, 137, 3683-3689

[6] Cowan, A. E.; Olivastro, E. M.; Koppel, D. E.; Loshon, C. A.; Setlow, B.; Setlow, P. Proc. Natl. Acad. Sci. USA 2004, 101, 7733-7738

[7] Cowan, A. E.; Koppel, D. E.; Setlow, B.; Setlow, P. Proc. Natl. Acad. Sci. USA 2003, 100, 4209-4214

[8] Leuschner, R. G. K.; Lillford, P. J. Int. J. Food Microbiol. 2001, 63, 35-50

[9] Popham, D.; Helin, J.; Costello, C.; Setlow, P. Proc. Natl. Acad. Sci. USA 1996, 93, 15405-15410

[10] Sahin, O.; Yong, E. H.; Driks, A.; Mahadevan, L. J. Roy. Soc. Interf. 2012, 9, 3156-3160

[11] Driks, A. Proc. Natl. Acad. Sci. USA 2003, 100, 3007-3009

[12] Westphal, A. J.; Price, P. B.; Leighton, T. J.; Wheeler, K. E. Proc. Natl. Acad. Sci. USA 2003, 100, 34613466

[13] Popham, D. L.; Helin, J.; Costello, C. E.; Setlow, P. J. Bact. 1996, 178, 6451-6458

[14] Thwaites, J. J.; Surana, U. C. J. Bact. 1991, 173, $197-$ 203

[15] Loison, P.; Hosny, N. A.; Gervais, P.; Champion, D.; Kuimova, M. K.; Perrier-Cornet, J.-M. BBA Biomembranes 2013

[16] Doster, W.; Cusack, S.; Petry, W. Nature 1989, 337, 754-756 
[17] Frauenfelder, H.; Parak, F. and Young, R. D. Annu. Rev. Biophys. Biophys. Chem. 1988, 17, 451-479

[18] Di Pace, A.; Cupane, A.; Leone, M.; Vitrano, W. and Cordone, L. Biophys. J. 1992, 63, 475-484

[19] Gabel, F.; Bicout, D.; Lehnert, U.; Tehei, M.; Weik, M. and Zaccai, G. Quart. Rev. Biophys. 2002, 35, 327-367

[20] Frölich, A.; Gabel, F.; Jasnin, M.; Lehnert, U.; Oesterhelt, D.; Stadler, A.M.; Tehei, M.; Weik, M.; Wood, K. and Zaccai, G. Faraday Discuss. 2009, 141, 117-130

[21] Schiró, G.; Caronna, C.; Natali, F.; Cupane, A. J Am Chem Soc. 2010, 132, 1371-1376

[22] Schiró, G.; Caronna, C.; Natali, F.; Koza, M. M.; Cupane, A. J. Phys. Chem. Lett. 2011, 2, 2275-2279

[23] Telling, M. T. F.; Clifton, L.; Combet, J.; Frick, B.; Howells, S.; Sakai, V. G. Soft Matt. 2012, 8, 95299532

[24] Pieper, J.; Buchsteiner, A.; Dencher, N. A.; Lechner, R. E.; Hauß, T. Phys Rev Lett. 2008, 100, 228103

[25] Combet, S.; Zanotti, J.-M. Phys. Chem. Chem. Phys. 2012, 14, 4927-4934

[26] Russo, D.; Teixeira, J.; Kneller, L.; Copley, J. R. D.; Ollivier, J.; Perticaroli, S.; Pellegrini, E.; Gonzalez, M. A. J Am Chem Soc. 2011, 133, 4882-4888

[27] Tehei, M.; Franzetti, B.; Madern, D.; Ginzburg, M.; Ginzburg, B. Z.; Giudici-Orticoni, M. T.; Bruschi, M.; Zaccai, G. EMBO reports 2004, 5, 66-70

[28] Mikl, C.; Peters, J.; Trapp, M.; Kornmueller, K.; Schneider, W.J. and Prassl, R. J. Am. Chem. Soc. 2011, 133, 13213-13215

[29] Falourd, X.; Natali, F.; Peters, J. and Foucat, L. Chem. Phys. 2014, 428, 181-185

[30] Natali, F.; Dolce, C.; Peters, J.; Gerelli, Y.; Stelletta, C. and Leduc, G. J. Phys. Soc. Jpn, 2013, 82, SA017

[31] Setlow, B.; Setlow, P. J. Bact. 1996, 178, 3486-3495

[32] Nicholson, W. L.; Setlow, P.; John Wiley \& Sons Ltd, Chichester, UK: 1990

[33] Natali, F.; Peters, J.; Russo, D.; Barbieri, S.; Chiapponi, C.; Cupane, A.; Deriu, A.; Di Bari,
M. T.; Farhi, E.; Gerelli, Y. Neutron News. 2008, 19, $14-18$

[34] Zaccai, G. Science. 2000, 288, 1604-1607

[35] Reat, V.; G. Zaccai; M. Ferrand; Pfister., C. In Biological Macromolecular Dynamics. S. Cusack, H. Büttner, M. Ferrand, P. Langan, and P Timmins, editors. Adenine Press, Schenectady, NY. 117-121, 1997

[36] Coleman, W. H.; Chen, D.; Li, Y.-Q.; Cowan, A. E.; Setlow, P. J. Bact. 2007, 189, 8458-8466

[37] Beaman, T. C.; Gerhardt, P. Appl. Env. Microbiol. 1986, 52, 1242-1246

[38] Wood, K; Grudinin, S.; Kessler, B.; Weik, M.; Johnson, M.; Kneller, G.R.; Oesterhelt, D. and Zaccai, G. J. Mol. Biol. 2008, 380, 581-591

[39] Peters, J.; Giudici-Orticoni, M.T.; Zaccai, G. and Guiral, M. Eur. Phys. J. E 2013, 36: 78

[40] Di Bari, M.; Deriu, A.; Albanese, G.; Cavatorta, F. Chem Phys. 2003, 292, 333-339

[41] Paciaroni, A.; Cinelli, S.; Onori, G. Biophys. J. 2002, 83, 1157-1164

[42] Minh, H. N. T.; Perrier-Cornet, J.-M.; Gervais, P.; Champion, D. Food Biophys. 2011, 5, 238-246

[43] Leuschner, R. G. K.; Lillford, P. J. Int. J Food Microbiol. 2003, 80, 131-143

[44] Belliveau, B. H.; Beaman, T. C.; Pankratz, H. S.; Gerhardt, P. J. Bact. 1992, 174, 4463-4474

[45] Sapru, V.; Labuza, T. P. J. Food Sci. 1993, 58, 445448

[46] Ablett, S.; Darke, A. H.; Lillford, P. J.; Martin, D. R. Int. J. Food Sci. Tech. 1999, 34, 59-69

[47] Cordone, L.; Ferrand, M.; Vitrano E.; Zaccai, G. Biophys. J. 76, 1043 (1999)

[48] Russo, D.; Pérez, J.; Zanotti, J.-M.; Desmadril, M.; Durand, D. Biophys. J. 2002, 83, 2792-2800

[49] Gibrat, G.; Assairi, F. L.; Blouquit, Y.; Craescu, C. T.; Bellissent-Funel, M.-C. Biophys J. 2008, 95, 52475256

[50] Receveur, V.; Calmettes, P.; Smith, J. C.; Desmadril, M.; Coddens, G.; Durand, D. Proteins. 1997, 28, 380-387 